\title{
Hydrophobic Interaction Chromatography for Bottom-Up Proteomics Analysis of Single Proteins and Protein Complexes
}

\author{
Michal Rackiewicz, ${ }^{\dagger, \ddagger}$ Ludger Große-Hovest, ${ }^{\S}$ Andrew J. Alpert, ${ }^{\|}$Mostafa Zarei, ${ }^{*, \perp}$ and Jörn Dengjel ${ }^{*}, \dagger(\$$ \\ ${ }^{\dagger}$ Department of Biology, University of Fribourg, Chemin du Musée 10, CH-1700 Fribourg, Switzerland \\ ${ }^{\ddagger}$ Department of Dermatology, Medical Center - University of Freiburg, Hauptstr. 7, D-79104 Freiburg, Germany \\ ${ }^{\S}$ SYNIMMUNE GmbH, Alte Landstrasse 42, D-72072 Tübingen, Germany \\ "PolyLC Inc., 9151 Rumsey Road, Columbia, Maryland 21045, United States \\ ${ }^{\perp}$ Solvias AG, Römerpark 2, CH-4303 Kaiseraugst, Switzerland
}

Supporting Information

\begin{abstract}
Hydrophobic interaction chromatography (HIC) is a robust standard analytical method to purify proteins while preserving their biological activity. It is widely used to study post-translational modifications of proteins and drug-protein interactions. In the current manuscript we employed HIC to separate proteins, followed by bottomup LC-MS/MS experiments. We used this approach to fractionate antibody species followed by comprehensive peptide mapping as well as to study protein complexes in human cells. HIC-reversed-phase chromatography (RPC)-mass spectrometry (MS) is a powerful alternative to fractionate proteins for bottom-up proteomics experiments making use of their distinct hydrophobic properties.
\end{abstract}

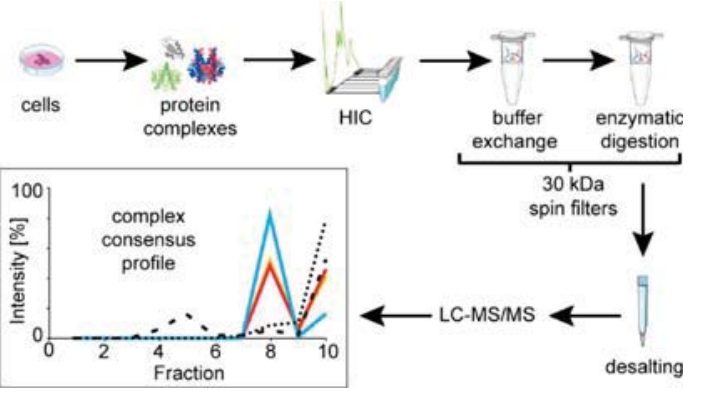

KEYWORDS: antibody, protein complex, chromatography, mass spectrometry, peptide, bottom-up proteomics, enzyme, biologic

\section{INTRODUCTION}

Knowledge of the proteome is critical for our understanding of pathophysiological processes. To study proteome alterations online liquid chromatography-tandem mass spectrometry (LC-MS/MS) is widely used. Recent technical advances allow for identification of $>10000$ proteins in a cancer cell line as well as whole yeast proteome characterizations within a few hours. ${ }^{1,2}$ To achieve such levels of proteome coverage, replicate analyses or a reduction in sample complexity, on the protein or peptide level, combined with multiple MS analyses is still critical. On the peptide level chromatography methods, like strong cation exchange (SCX) and hydrophilic interaction chromatography (HILIC), as well as high-pH reversed phase chromatography have been employed successfully. ${ }^{3-6}$ Because of its robustness and ease of handling, the classical and still widely used approach for protein fractionation prior to LCMS/MS is gel-based separation under denaturing conditions (SDS-PAGE). ${ }^{7}$ Recently, size exclusion chromatography (SEC) has been employed to elucidate native protein complexes based on coelution profiles. ${ }^{8-11}$

For the detailed characterization of single proteins, hydrophobic interaction chromatography (HIC) has emerged as one of the key bioanalytical methods. ${ }^{12}$ HIC is a high-resolution chromatography mode based on the interaction of weakly hydrophobic ligands of the stationary phase with hydrophobic patches on the surface of the tertiary structure of proteins. ${ }^{13,14}$ By employment of high concentrations of structure-promoting ("kosmotropic") salts, proteins in HIC retain their conforma- tional structure. ${ }^{15}$ Proteins are eluted in order of increasing hydrophobicity with a gradient of decreasing ionic strength of the mobile phase. The high sensitivity of the method allows distinction between isomers and conformational states of therapeutic proteins. ${ }^{16-18}$ Recent advances have highlighted the compatibility of HIC with direct MS analysis. Online HICMS as well as HIC-RPC-MS have been successfully employed for top-down proteomics analyses of single proteins and complex protein mixtures. 19,20

We considered HIC to be a promising alternative to already established fractionation techniques for studying single proteins and protein complexes by bottom-up MS-based proteomics. The developed workflow is suitable for characterization of native soluble protein complexes as well as peptide mapping of single purified proteins and biologics.

\section{EXPERIMENTAL SECTION}

Chemicals and Reagents

All reagents were purchased from Sigma-Aldrich (Munich, Germany) unless noted otherwise.

\section{Cell Culture}

$\mathrm{HeLa}$ and CaCo-2 cells were cultured in Dulbecco's modified Eagle's medium (DMEM, Thermo Fisher Scientific, Langenselbold, Germany) supplemented with $10 \%$ fetal bovine serum 
A
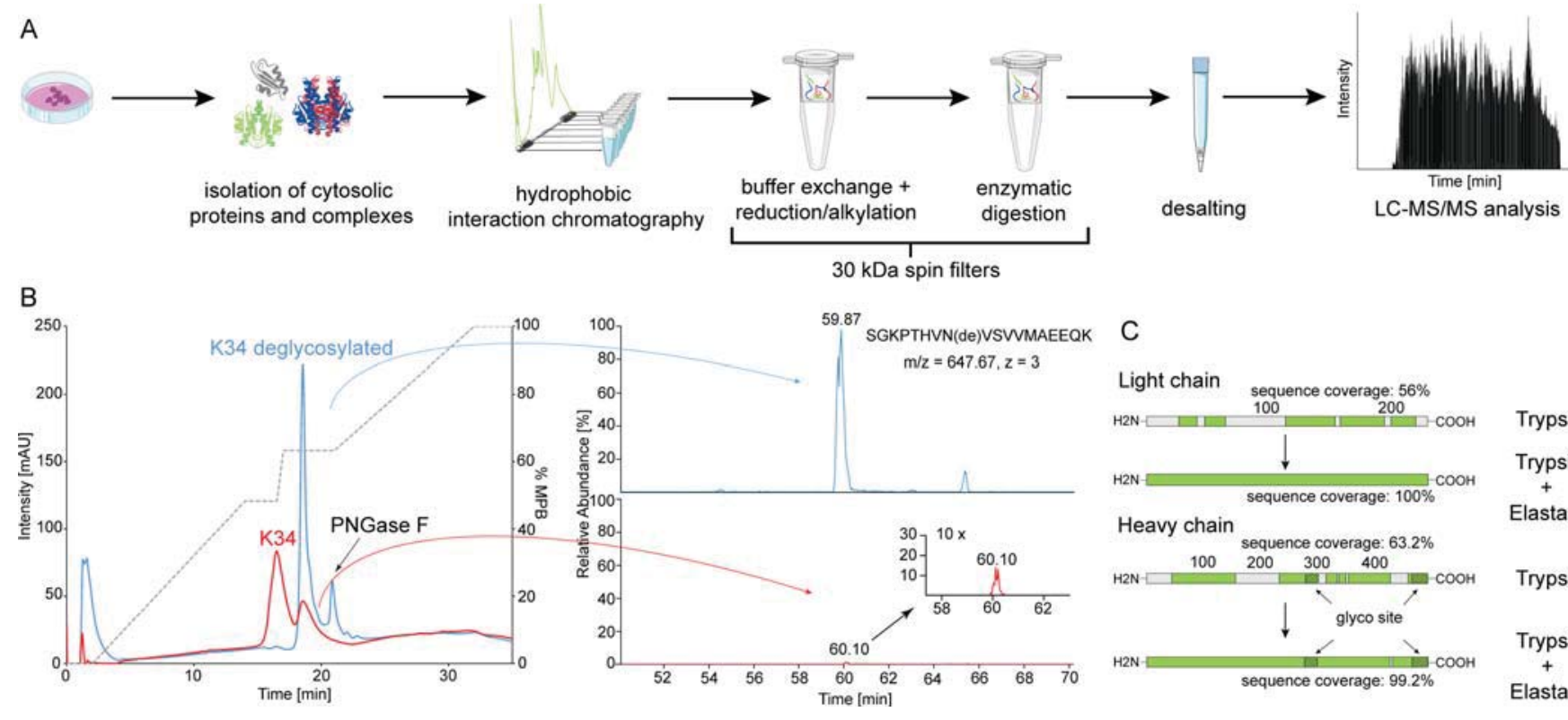

$30 \mathrm{kDa}$ spin filters

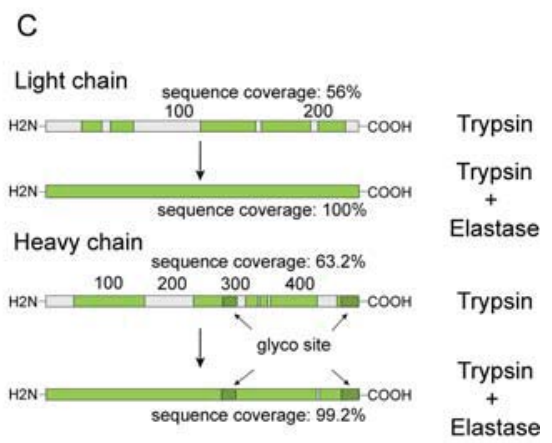

Figure 1. Workflow and analysis of antibody modifications. (A) HIC-RPC-MS workflow. Cells are lysed using a dounce homogenizer and cytosol is enriched by a centrifugation step to remove cellular membranes. Cytosol ( $3 \mathrm{mg}$ protein) is diluted in HIC buffer A, and proteins are separated by HIC. Per 30 min HIC run, 20 fractions are collected. Each fraction is transferred to MWCO filters of $30 \mathrm{kDa}$ ( $3 \mathrm{kDa}$ in the case of antibody), and buffer is exchanged to $1 \% \mathrm{NaDOC}$ in $\mathrm{ABC}$ buffer. Proteins are reduced, alkylated, and digested by trypsin. The generated peptides are filtered and desalted by STAGE tips prior to LC-MS/MS analysis. (B) Analysis of antibody modifications. The chimeric antibody K34 was resolved by HIC into two peaks (shown in red, left part of panel; HIC gradient is indicated by dotted black line). After PNGase F treatment the first peak disappeared, yielding a single antibody peak shown in blue, indicating that the first peak contained N-linked glycan moieties. Antibody fractions were processed as indicated in panel A, and the glycosylation sites were mapped to residues N298 and N456, respectively. Respective mass chromatograms for peptides containing the N456 glycosylation site are shown in the right part of the panel. The deglycosylated peptide was 100 times more abundant after PNGase F treatment. (C) Antibody sequence coverage. Whereas HIC in combination with tryptic digestion yielded $60 \%$ antibody sequence coverage, HIC in combination with both trypsin and elastase yielded close to $100 \%$ sequence coverage.

(Gibco, Darmstadt, Germany), 1\% L-glutamine, and 1\% PenStrep (both from PAN Biotech, Aidenbach, Germany). Cells were cultured in humidified air supplemented with $5 \% \mathrm{CO}_{2}$ at $37{ }^{\circ} \mathrm{C}$.

\section{Sample Preparation}

Cell pellets of three $15 \mathrm{~cm}$ cell culture dishes were suspended in $2 \mathrm{~mL}$ of lysis buffer (50 mM NaOAc, $50 \mathrm{mM} \mathrm{KCl}$ (Merck, Darmstadt, Germany) with the addition of $1 \times$ protease and phosphatase inhibitors (Roche, Penzberg, Germany) and homogenized for $5 \mathrm{~min}$ on ice by douncing. Cell lysate was cleared of cell debris and nuclei by centrifugation at $4{ }^{\circ} \mathrm{C}, 3220 \mathrm{~g}$ for $20 \mathrm{~min}$, followed by $4{ }^{\circ} \mathrm{C}$ for $20 \mathrm{~min}$ at $21000 \mathrm{~g}$. The pellet was discarded, and cell lysates were concentrated on a $100 \mathrm{kDa}$ molecular weight cutoff filter (Sartorius, Göttingen, Germany). Protein concentration was determined with a BCA assay kit (Thermo Fisher, Bremen, Germany). The supernatant solution containing $3 \mathrm{mg}$ of cytosolic protein complexes was diluted 1:1 (V/V) with 2 M HIC mobile phase A and used directly for HIC. Fc-optimized chimeric Ig $\gamma 1$ antibody K34 was deglycosylated with PNGase $\mathrm{F}$ at $37^{\circ} \mathrm{C}$, shaking overnight. Control K34 samples were incubated accordingly without PNGase F.

\section{Hydrophobic Interaction Chromatography}

A General Electric ÄKTA 900 HPLC system (GE Healthcare, Freiburg, Germany) equipped with a PolyPROPYL A column $(3 \mu \mathrm{m}, 1500 \AA$ A $100 \mathrm{~mm} \times 4.6 \mathrm{~mm}$ i.d.; PolyLC, Columbia, MD) was employed for HIC separation. Mobile phase A (MPA) and mobile phase B (MPB) containing either $2 \mathrm{M}$ and $20 \mathrm{mM}$ ammonium tartrate (AT) or the same amount of ammonium sulfate (AS), respectively, were adjusted to $\mathrm{pH} 7.0$ with $10 \% \mathrm{NH}_{4} \mathrm{OH}$. Mobile phases were filtered on a $0.22 \mu \mathrm{m}$ vacuum filter (Corning, Corning, NY) before use.

For cytosol, a 30 min linear gradient from $100 \%$ MPA to $100 \% \mathrm{MPB}$, followed by $5 \mathrm{~min}$ of $100 \% \mathrm{MPB}$ and $100 \% \mathrm{MPA}$ for $5 \mathrm{~min}$, was employed at a flow rate of $1 \mathrm{~mL} / \mathrm{min}$. For the K34 antibody samples the linear gradient was interrupted with two isocratic regions at $48.3 \% \mathrm{MPB}(12-14.2 \mathrm{~min})$ and $63.3 \%$ MPB (15-19 min). Fractions were collected one per minute throughout the gradient. Absorbance was measured at $\lambda=280$ $\mathrm{nm}$.

\section{Filter-Based Sample Preparation for LC-MS/MS Analysis}

Neighboring HIC fractions of whole cell lysates were pooled to give 10 final fractions. Samples were loaded onto $30 \mathrm{kDa}$ MWCO filters (PALL, Basel, Switzerland; $3 \mathrm{kDa}$ (Sartorius) for the $\mathrm{K} 34$ antibody) and washed with three times $500 \mu \mathrm{L}$ of $1 \%$ sodium deoxycholate (NaDOC)/0.1 M ammonium bicarbonate $(\mathrm{ABC})$. Proteins were reduced/alkylated with $5 \mathrm{mM}$ tris (2carboxyethyl)-phosphine (TCEP)/5.5 mM chloroacetamide (30 min, RT) before trypsin (Promega, Mannheim, Germany) and LysC (Wako, Neuss, Germany) digestion (each 1:100 w/ w) overnight at $37^{\circ} \mathrm{C}$. Peptides were filtered and acidified with $1 \%$ trifluoroacetic acid (TFA), NaDOC was removed by centrifugation, and supernatants containing peptides were desalted by STAGE tips as described. ${ }^{21} \mathrm{~K} 34$ antibody samples were digested with trypsin for $4 \mathrm{~h}$, followed by $2 \mathrm{~h}$ of elastase (Promega) in ABC buffer at $37^{\circ} \mathrm{C}$. Samples were evaporated to $5 \mu \mathrm{L}$ and acidified with $10 \mu \mathrm{L}$ of $3 \% \mathrm{ACN} / 0.3 \%$ TFA (both from LGC Promochem, Wesel, Germany). 


\section{In-Gel Digestion}

An aliquot of $350 \mu \mathrm{g}$ protein from the cytosolic cell fraction was incubated for $10 \mathrm{~min}$ at $95{ }^{\circ} \mathrm{C}$ in SDS-PAGE loading buffer, reduced, and alkylated as described. Protein mixtures were separated by SDS-PAGE using 4-12\% NuPAGE gels (Invitrogen, Darmstadt, Germany). Gel lanes were cut into 10 equal slices, and proteins therein were in-gel-digested with trypsin overnight at $37^{\circ} \mathrm{C}$. ${ }^{7}$ Resulting peptides were desalted on STAGE tips. $^{21}$

\section{LC-MS/MS}

Mass spectrometric measurements were performed on a LTQ Orbitrap XL mass spectrometer (Thermo Fisher Scientific) coupled to an Agilent 1200 nanoflow-HPLC (Agilent Technologies, Waldbronn, Germany). HPLC-column tips (fused silica) with $75 \mu \mathrm{m}$ inner diameter (New Objective, Woburn, MA) were self-packed with Reprosil-Pur 120 ODS-3 (Dr. Maisch, Ammerbuch, Germany) to a length of $20 \mathrm{~cm}$. Samples were applied directly onto the column without precolumn. A gradient of A ( $0.5 \%$ acetic acid (LGC Promochem) in water and $\mathrm{B}(0.5 \%$ acetic acid in $80 \%$ ACN (LC-MS grade, Wako) in water) with increasing organic proportion was used for peptide separation (loading of sample with $2 \% \mathrm{~B}$; separation ramp: from $10-30 \% \mathrm{~B}$ within $80 \mathrm{~min}$ ). The flow rate was 250 $\mathrm{nL} / \mathrm{min}$ and for sample application $500 \mathrm{~nL} / \mathrm{min}$. The mass spectrometer was operated in the data-dependent mode and switched automatically between MS (max. of $1 \times 10^{6}$ ions) and MS/MS. Each MS scan was followed by a maximum of five MS/MS scans in the linear ion trap using normalized collision energy of $35 \%$ and a target value of 5'000. Parent ions with a charge state of $z=1$ and unassigned charge states were excluded from fragmentation. The mass range for MS was $m / z$ $=370-2000$. The resolution was set to 60000 . Massspectrometric parameters were as follows: spray voltage 2.3 $\mathrm{kV}$; no sheath and auxiliary gas flow; and ion-transfer tube temperature $200{ }^{\circ} \mathrm{C}$.

The mass spectrometry proteomics data have been deposited to the ProteomeXchange Consortium via the PRIDE partner repository $^{22}$ with the data set identifier PXD006217, Project Name: Hydrophobic interaction chromatography for bottomup proteomics.

\section{Data Analysis and Statistics}

Raw MS data files were processed using MaxQuant version 1.4.1.2, ${ }^{23}$ with a false discovery rate $($ FDR $)<0.01$ at the level of proteins, peptides, and modifications, using default settings with the following minor changes: oxidized methionine (M) and acetylation (protein N-term) and deamidation (NQ) (only for antibody analysis) as variable modifications and carbamidomethyl (C) as fixed modification, minimum peptide length of seven amino acids, and "match between runs" (MBR) enabled with a matching time window of $1 \mathrm{~min}$. Proteins and peptides were identified using a target-decoy approach with a reversed database using the Andromeda search engine integrated into the MaxQuant environment. ${ }^{24}$ Searches were performed against the Human UniProt FASTA database (July 2015), and quantification of peptides and proteins was performed by MaxQuant. Bioinformatics analysis was performed with MultiExperiment Viewer (http://mev.tm4.org/), Perseus, and Microsoft Excel.

\section{RESULTS AND DISCUSSION}

\section{HIC Analysis of K34 Antibody and MS Peptide Mapping}

To establish a HIC-based workflow suited for bottom-up proteomics MS analysis (Figure 1A) a set of standard proteins was used (Figure S1). We used a spin-filter-based approach to exchange HIC buffers to allow proteolytic digestion for bottomup proteomics MS analyses. ${ }^{25}$ Structure-promoting ammonium salts were replaced by three consecutive washing steps with $1 \%$ $\mathrm{NaDOC}$ in $0.1 \mathrm{M}$ ammonium bicarbonate (ABC). After reduction and alkylation of cysteine side chains, proteins were digested on filter by trypsin. The resulting peptide mixtures were filtered and acidified to deplete NaDOC (see Experimental Section for details). Finally, peptides were desalted by STAGE tips and analyzed by LC-MS/MS. After achieving satisfactory results with standard proteins (data not shown), we applied our workflow to the Fc-optimized chimeric $\operatorname{Ig} \gamma 1$ antibody $\mathrm{K} 34$.

The HIC chromatogram of the K34 antibody showed two peaks, which were not baseline-separated, at $17^{\prime}$ (peak I) and $19^{\prime}$ (peak II), indicating two distinct variants (Figure 1B, left panel, red chromatogram). The Fc-optimized chimeric antibody $\mathrm{K} 34$ has, in addition to the glycosylation site of human $\operatorname{Ig} \gamma 1$ isotypes at N297, a second glycosylation site at N456 in the C-terminal part of its heavy chain. Indeed, after PNGase F treatment (Figure S2) only the second peak at $19 \mathrm{~min}$ remained, being more intense than before the treatment and indicating that peak I represented the glycosylated variant of K34 (Figure 1B, left panel, blue chromatogram). Tryptic digests from both peaks were analyzed by LC-MS/MS, yielding a protein coverage of $56 \%$ for the light chain and $63.2 \%$ for the heavy chain, respectively (Figure 1C). To unambiguously pinpoint the glycosylation sites, proteolytic digestion protocols were optimized to yield optimal sequence coverage. A consecutive proteolytic digestion protocol combing on-filtertrypsin and -elastase digestion drastically improved sequence coverage of both light (100\%) and heavy chains (99.2\%). Using the newly established protocol we were able to localize the glycosylation sites to N297 and N456 of the antibody heavy chain, the amino acid residues lying within N-glycosylation consensus sequence motifs $\mathrm{N}-\mathrm{X}-\mathrm{S} / \mathrm{T}$ (with $\mathrm{X}$ being any amino acid except proline). The peptide containing the deglycosylated (de) N456 residue "SGKPTHVN(de)VSVVMAEEQK" showed a 100-fold increase in intensity after PNGase $F$ treatment (Figure 1B, right panel), the peptide "EEQYN(de)STYR” containing N297 a 200-fold increase (Figure S3). Additionally, changes in oxidation status were ruled out. Thus using HIC plus bottom-up proteomics we comprehensively characterized the antibody K34 including two glycosylation sites at N297 and N456.

\section{HIC-based Characterization of Cytosolic Protein Complexes}

Virtually all cellular processes driving cell proliferation, growth, and homeostasis depend on the dynamic regulation of physical protein-protein interactions. Thus the study of protein complexes is critical for the understanding of pathophysiological processes. Whereas protein separation by SDS-PAGE has been widely and successfully used to establish cellular protein inventories, ${ }^{26}$ knowledge about protein interactions is lost due to the denaturing conditions during sample preparation. We used the established HIC-bottom-up proteomics workflow to analyze cytosolic protein complexes and 
A

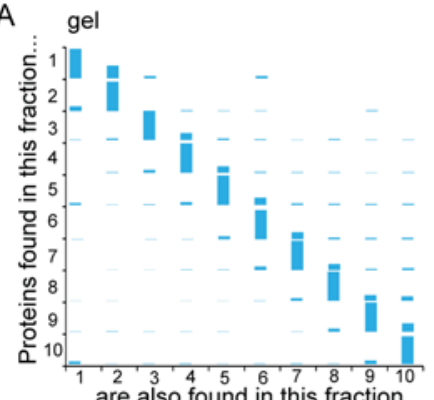

HIC

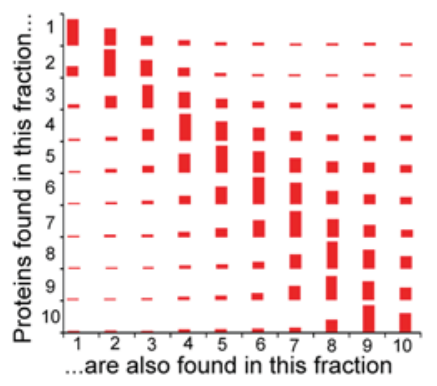

E
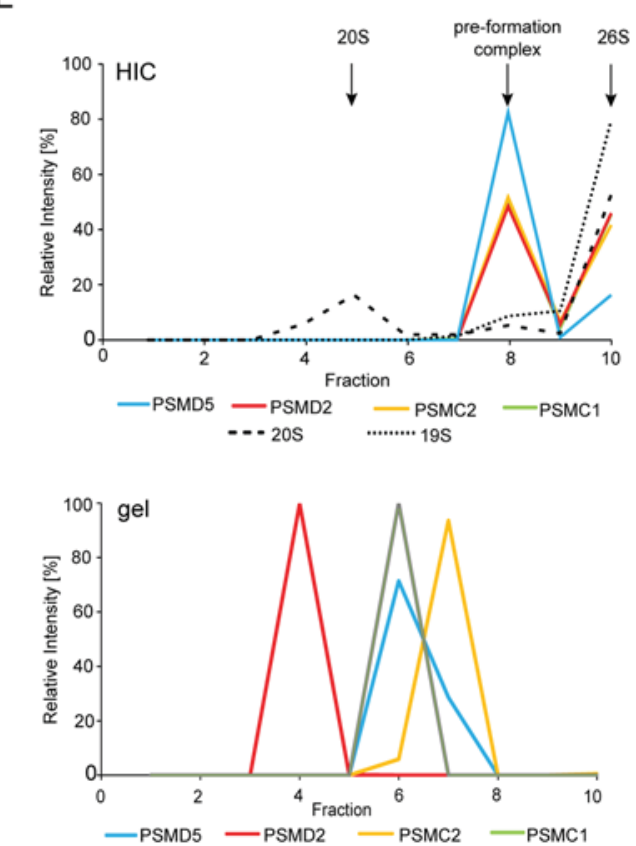

C

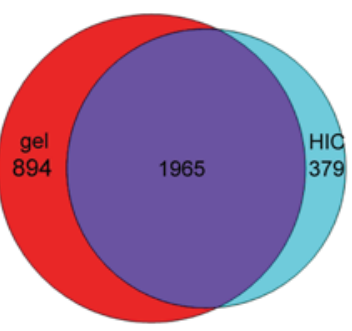

D

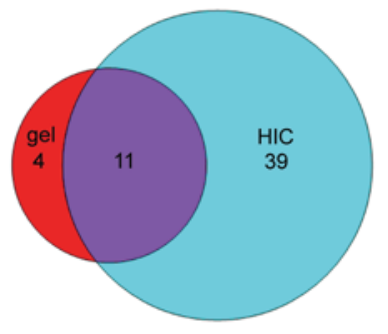

$\mathrm{F}$

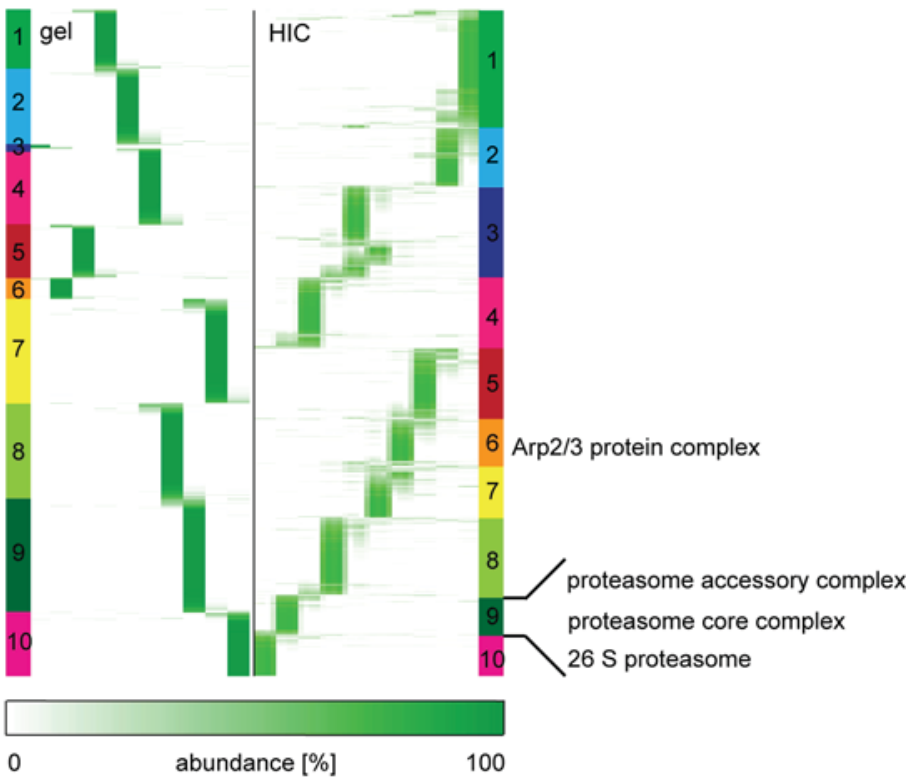

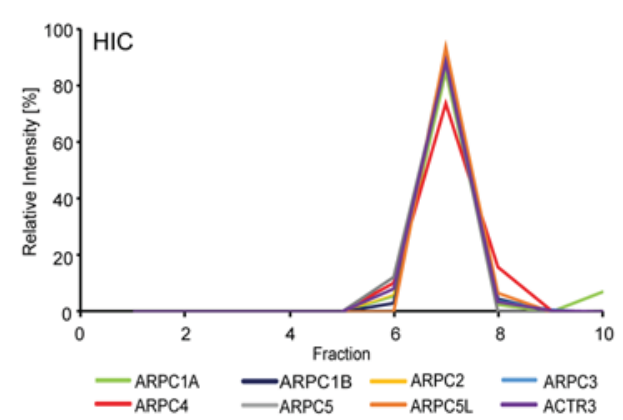

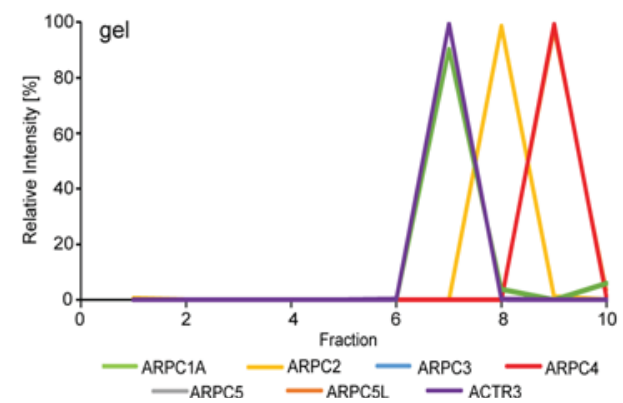

Figure 2. Analysis of protein complexes by HIC. (A) Comparison of HIC with geLC-MS. Whereas prefractionation by SDS-PAGE separated proteins according to size, HIC separated proteins according to hydrophobicity. Thus in geLC-MS proteins commonly localize to single gel fractions. By contrast, distinct protein complexes of specific proteins were found in several HIC fractions. (B) Protein identifications. Venn diagram comparing protein identifications by geLC-MS and HIC-RPC-MS. (C) Cluster analysis of protein profiles. Relative distributions of proteins fractionated by SDS-PAGE and HIC were clustered using k-means. Enrichment analysis using the CORUM annotations yielded enriched protein complex terms in HIC but not in gel clusters (see Supplemental Tables S2 and S3 for complete lists). (D) Enriched CORUM terms. Venn diagram comparing geLC-MS and HIC-RPC-MS approaches by enriched CORUM terms in respective cluster analyses shown in panel C. (E) Proteasome protein profiles. Whereas SDS-PAGE separated proteins from the proteasomal preformation complex into several fractions according to their size, HIC yielded a preformation complex consensus profile. This overlapped partially with the profiles of the $26 \mathrm{~S}$ proteasome complexes (indicated by dotted lines). (F) Arp $2 / 3$ protein profiles. Similarly, as shown in panel E, Arp2/3 proteins eluted as a unitary complex in HIC but were separated in different fractions by SDS-PAGE. Note: geLC-MS analyses were performed with HeLa cell lysate. HIC-RPC-MS analyses were performed with both CaCo-2 cell lysate shown here and HeLa cell lysate shown in Figure S4.

compared the generated data to classical gel-based bottom-up proteomics analyses.

Whereas the separation by size in SDS-PAGE leads to the focusing of proteins into single gel bands, HIC leads to a broader distribution of proteins (Figure $2 \mathrm{~A}$ ). More than $66 \%$ of proteins are found in single bands in SDS-PAGE, but only $24 \%$ of proteins elute in single HIC fractions. This broader distribution is very likely due to participation of proteins in multiple complexes (see below). The broader distribution and thus less relative concentration of proteins may also be the 
reason for lower identification rates in HIC-MS compared with SDS-PAGE-MS (Figure 2B and Supplemental Table S1). Critically, separation by HIC is robust and cell-lineindependent. Cytosol fractionations of $\mathrm{HeLa}$ and $\mathrm{CaCo}-2$ cell samples gave very similar results (Figure 2 and Figure S4).

To elucidate the suitability of HIC for the analysis of protein complexes by bottom-up MS-based proteomics, we clustered elution profiles and performed bioinformatics enrichments analyses on respective clusters using the CORUM annotation of protein complexes (Figure 2C). ${ }^{27}$ As anticipated, we identified significantly more enriched complexes in HIC compared with SDS-PAGE analyses (Figure 2D, $p<0.05$, BH-corrected; Supplemental Tables S2 and S3), two examples being the proteasome and the Arp $2 / 3$ protein complex. Proteins PSMC1 and PSMC2 together with PSMD2 and PSMD5 are known to associate in a transient preformation complex of the $26 \mathrm{~S}$ proteasome. ${ }^{28,29}$ In SDS-PAGE, proteins distributed in several gel bands according to their size (Figure $2 \mathrm{E}$ ). In HIC two peaks containing all of the proteins were observed: The second peak eluted together with other members of the PSMA, PSMB, PSMC, and PSMD protein families, indicating that this peak corresponds to the $26 \mathrm{~S}$ proteasome. Thus HIC was able to separate the 20S, 26S proteasome and the preformation complex. Also, the Arp2/3 complex eluted as a unit in HIC, whereas its constituents were separated by size in SDS-PAGE (Figure 2F).

\section{CONCLUSIONS}

Taken together, HIC in combination with bottom-up proteomics allows peptide mapping and detailed post-translational modification (PTM) analysis of single proteins as well as the study of protein complexes and interactions. Because the mode of separation is based on hydrophobicity and not on size, as per SDS-PAGE and SEC, this approach will be particularly useful for studying the role of PTMs in the formation of complexes as well as drug-protein interactions.

\section{ASSOCIATED CONTENT}

\section{Supporting Information}

The Supporting Information is available free of charge on the ACS Publications website at DOI: 10.1021/acs.jproteome.7b00015.

Figure S1: HIC analyses of standard proteins. Figure S2: HIC analyses of PNGase F. Figure S3: Mass chromatograms of K34 antibody peptides containing the N298 glycosylation site. Figure S4: HIC-RPC-MS analyses of HeLa cell cytosol. (PDF)

Supplemental Table S1: Protein identifications by SDSPAGE and HIC. (XLSX)

Supplemental Table S2: Enriched CORUM terms from HIC analysis. (XLSX)

Supplemental Table S3: Enriched CORUM terms from SDS-PAGE analysis. (XLSX)

\section{AUTHOR INFORMATION}

\section{Corresponding Authors}

*M.Z.: E-mail: Mostafa.Zarei@SOLVIAS.com. Tel: +41 61845 6563.

*J.D.: E-mail: joern.dengjel@unifr.ch. Tel: +41 263008631. Fax: +41263009741.

\section{ORCID}

Jörn Dengjel: 0000-0002-9453-4614 Notes

The authors declare the following competing financial interest(s): A.J.A. works for the company that supplied the HIC column.

The mass spectrometry proteomics data have been deposited to the ProteomeXchange Consortium via the PRIDE partner repository ${ }^{22}$ with the data set identifier PXD006217, Project Name: Hydrophobic interaction chromatography for bottomup proteomics.

\section{ACKNOWLEDGMENTS}

The research leading to these results has received funding from the Swiss National Science Foundation, grant 31003A-166482/ 1 (J.D.).

\section{REFERENCES}

(1) Hebert, A. S.; Richards, A. L.; Bailey, D. J.; Ulbrich, A.; Coughlin, E. E.; Westphall, M. S.; Coon, J. J. The one hour yeast proteome. Mol. Cell. Proteomics 2014, 13 (1), 339-47.

(2) Nagaraj, N.; Wisniewski, J. R.; Geiger, T.; Cox, J.; Kircher, M.; Kelso, J.; Paabo, S.; Mann, M. Deep proteome and transcriptome mapping of a human cancer cell line. Mol. Syst. Biol. 2011, 7, 548.

(3) Batth, T. S.; Olsen, J. V. Offline High pH Reversed-Phase Peptide Fractionation for Deep Phosphoproteome Coverage. Methods Mol. Biol. 2016, 1355, 179-92.

(4) Chen, W.; Wang, S.; Adhikari, S.; Deng, Z.; Wang, L.; Chen, L.; Ke, M.; Yang, P.; Tian, R. Simple and Integrated Spintip-Based Technology Applied for Deep Proteome Profiling. Anal. Chem. 2016, 88 (9), 4864-71.

(5) Zarei, M.; Sprenger, A.; Rackiewicz, M.; Dengjel, J. Fast and easy phosphopeptide fractionation by combinatorial ERLIC-SCX solidphase extraction for in-depth phosphoproteome analysis. Nat. Protoc. 2015, 11 (1), 37-45.

(6) Zhou, H.; Di Palma, S.; Preisinger, C.; Peng, M.; Polat, A. N.; Heck, A. J.; Mohammed, S. Toward a comprehensive characterization of a human cancer cell phosphoproteome. J. Proteome Res. 2013, 12 (1), 260-71.

(7) Shevchenko, A.; Tomas, H.; Havlis, J.; Olsen, J. V.; Mann, M. Ingel digestion for mass spectrometric characterization of proteins and proteomes. Nat. Protoc 2007, 1 (6), 2856-60.

(8) Kristensen, A. R.; Gsponer, J.; Foster, L. J. A high-throughput approach for measuring temporal changes in the interactome. Nat. Methods 2012, 9 (9), 907-9.

(9) Park, S. J.; Ahn, H. S.; Kim, J. S.; Lee, C. Evaluation of MultitRNA Synthetase Complex by Multiple Reaction Monitoring Mass Spectrometry Coupled with Size Exclusion Chromatography. PLoS One 2015, 10 (11), e0142253.

(10) Wan, C.; Borgeson, B.; Phanse, S.; Tu, F.; Drew, K.; Clark, G.; Xiong, X.; Kagan, O.; Kwan, J.; Bezginov, A.; Chessman, K.; Pal, S.; Cromar, G.; Papoulas, O.; Ni, Z.; Boutz, D. R.; Stoilova, S.; Havugimana, P. C.; Guo, X.; Malty, R. H.; Sarov, M.; Greenblatt, J.; Babu, M.; Derry, W. B.; Tillier, E. R.; Wallingford, J. B.; Parkinson, J.; Marcotte, E. M.; Emili, A. Panorama of ancient metazoan macromolecular complexes. Nature 2015, 525 (7569), 339-44.

(11) Diedrich, B.; Rigbolt, K. T.; Roring, M.; Herr, R.; KaeserPebernard, S.; Gretzmeier, C.; Murphy, R. F.; Brummer, T.; Dengjel, J. Discrete cytosolic macromolecular BRAF complexes exhibit distinct activities and composition. EMBO J. 2017, 36 (5), 646-63.

(12) O'Connor, B. F.; Cummins, P. M. Hydrophobic Interaction Chromatography. Methods Mol. Biol. 2017, 1485, 355-63.

(13) Hjertén, S. Some general aspects of hydrophobic interaction chromatography. Journal of Chromatography A 1973, 87 (2), 325-31.

(14) Hjertén, S.; Rosengren, J.; Påhlman, S. Hydrophobic interaction chromatography. Journal of Chromatography A 1974, 101 (2), 281-8. 
(15) Alpert, A. J. High-performance hydrophobic-interaction chromatography of proteins on a series of poly(alkyl aspart-amide)silicas. Journal of Chromatography A 1986, 359, 85-97.

(16) Boyd, D.; Kaschak, T.; Yan, B. HIC resolution of an IgG1 with an oxidized $\operatorname{Trp}$ in a complementarity determining region. J. Chromatogr. B: Anal. Technol. Biomed. Life Sci. 2011, 879 (13-14), 955-60.

(17) Grebenau, R. C.; Goldenberg, D. M.; Chien-Hsing, C.; Koch, G. A.; Gold, D. V.; Kunz, A.; Hansen, H. J. Microheterogeneity of a purified IgG1 due to asymmetric Fab glycosylation. Mol. Immunol. 1992, 29 (6), 751-8.

(18) Wakankar, A. A.; Borchardt, R. T.; Eigenbrot, C.; Shia, S.; Wang, Y. J.; Shire, S. J.; Liu, J. L. Aspartate isomerization in the complementarity-determining regions of two closely related monoclonal antibodies. Biochemistry 2007, 46 (6), 1534-44.

(19) Chen, B.; Peng, Y.; Valeja, S. G.; Xiu, L.; Alpert, A. J.; Ge, Y. Online Hydrophobic Interaction Chromatography-Mass Spectrometry for Top-Down Proteomics. Anal. Chem. 2016, 88 (3), 1885-91.

(20) Xiu, L.; Valeja, S. G.; Alpert, A. J.; Jin, S.; Ge, Y. Effective protein separation by coupling hydrophobic interaction and reverse phase chromatography for top-down proteomics. Anal. Chem. 2014, 86 (15), 7899-906.

(21) Rappsilber, J.; Mann, M.; Ishihama, Y. Protocol for micropurification, enrichment, pre-fractionation and storage of peptides for proteomics using StageTips. Nat. Protoc. 2007, 2 (8), 1896-906.

(22) Vizcaino, J. A.; Csordas, A.; del-Toro, N.; Dianes, J. A.; Griss, J.; Lavidas, I.; Mayer, G.; Perez-Riverol, Y.; Reisinger, F.; Ternent, T.; Xu, Q. W.; Wang, R.; Hermjakob, H. 2016 update of the PRIDE database and its related tools. Nucleic Acids Res. 2016, 44 (D1), D447-56.

(23) Cox, J.; Mann, M. MaxQuant enables high peptide identification rates, individualized p.p.b.-range mass accuracies and proteome-wide protein quantification. Nat. Biotechnol. 2008, 26 (12), 1367-72.

(24) Cox, J.; Neuhauser, N.; Michalski, A.; Scheltema, R. A.; Olsen, J. V.; Mann, M. Andromeda: a peptide search engine integrated into the MaxQuant environment. J. Proteome Res. 2011, 10 (4), 1794-805.

(25) Wisniewski, J. R.; Zougman, A.; Nagaraj, N.; Mann, M. Universal sample preparation method for proteome analysis. Nat. Methods 2009, 6 (5), 359-62.

(26) Sprenger, A.; Weber, S.; Zarai, M.; Engelke, R.; Nascimento, J. M.; Gretzmeier, C.; Hilpert, M.; Boerries, M.; Has, C.; Busch, H.; Bruckner-Tuderman, L.; Dengjel, J. Consistency of the proteome in primary human keratinocytes with respect to gender, age, and skin localization. Mol. Cell. Proteomics 2013, 12 (9), 2509-21.

(27) Ruepp, A.; Waegele, B.; Lechner, M.; Brauner, B.; DungerKaltenbach, I.; Fobo, G.; Frishman, G.; Montrone, C.; Mewes, H. W. CORUM: the comprehensive resource of mammalian protein complexes-2009. Nucleic Acids Res. 2010, 38 (Database issue), D497-501.

(28) Kaneko, T.; Hamazaki, J.; Iemura, S.; Sasaki, K.; Furuyama, K.; Natsume, T.; Tanaka, K.; Murata, S. Assembly pathway of the Mammalian proteasome base subcomplex is mediated by multiple specific chaperones. Cell 2009, 137 (5), 914-25.

(29) Roelofs, J.; Park, S.; Haas, W.; Tian, G.; McAllister, F. E.; Huo, Y.; Lee, B. H.; Zhang, F.; Shi, Y.; Gygi, S. P.; Finley, D. Chaperonemediated pathway of proteasome regulatory particle assembly. Nature 2009, 459 (7248), 861-5. 